\title{
Orbital phase spectroscopy of GX 301-2 with RXTE-PCA
}

\author{
U. Mukherjee and B. Paul
}

\author{
Department of Astronomy \& Astrophysics, Tata Institute of Fundamental Research, Homi Bhabha Road, Colaba, \\ Mumbai-400 005, India \\ e-mail: uddipan;bpaul@tifr.res.in
}

Received 26 September 2003 / Accepted 29 June 2004

\begin{abstract}
We have investigated the orbital phase dependence of the X-ray spectrum of the High Mass X-ray Binary pulsar GX 301-2. Here we present the results from a spectral analysis of two sets of observations of GX 301-2 with the Rossi X-ray Timing Explorer (RXTE). Of particular interest are the variations of the absorption column density and the iron line flux along with other parameters of the spectral model with the orbital phase. We found that the X-ray spectrum can almost always be fitted with a partial covering absorption model. We have detected enhanced absorption near the periastron. However, the column density variation with orbital phase is not smooth, as is expected in a smooth stellar wind model. We discuss the results of the column density variation in the light of the two proposed models for GX 301-2, an equatorial disk emanating from the companion star Wray 977 and a gas stream following the neutron star. We also found that the iron $\mathrm{K} \alpha$ and $\mathrm{K} \beta$ line fluxes have peaks near the periastron and are well correlated with the continuum hard X-ray flux. The line equivalent width shows an interesting pattern with the column density, reasonably constant for low values of the column density and increasing rapidly beyond a certain value.
\end{abstract}

Key words. stars: pulsars: individual: GX 301-2 - stars: circumstellar matter - X-rays: stars

\section{Introduction}

In High Mass X-ray Binaries (HMXBs), the mass accretion onto the compact object can be from both the stellar wind and an outflowing equatorial disk of the companion star. Variation in the stellar wind accretion rate can give rise to a smooth modulation in the X-ray intensity with orbital phase, whereas strong enhancement in X-ray luminosity can be seen following the passage of the compact star through a circumstellar disk. If the companion star has a circumstellar disk, the occasional increase of material in the disk or an enhancement in the radial extent of the disk causes the strong X-ray outbursts in such systems. In many of the HMXBs, wind accretion plays a major role and the compact object is X-ray bright throughout the binary orbit, whereas in some other sources there is very little accretion from the stellar wind and transient accretion from the disk is more important. There are however a few systems like GX 301-2, in which a smoothly varying accretion component from the stellar wind and a rapidly varying component either from a disk or a trailing gas stream are important and present in almost all the binary orbits. A majority of the HMXBs harbour high magnetic field neutron stars causing the X-rays from the compact object to pulsate. X-ray irradiation and photoionisation of the stellar wind is very important in HMXBs. Measurement of the orbital evolution of the spectrum by observations in multiple orbital phases allows us to understand the wind structure and the effect of the wind on the X-ray spectrum both in terms of absorption and changing mass accretion rate. The presence and role of a circumstellar disk of the companion star in producing the orbital intensity variations in the light curve and changes in the absorption column density with the binary phase can also be investigated with multi phase observations of such systems.

GX 301-2 (4U 1223-62) is an HMXB pulsar (White et al. 1976) with an eccentric binary orbit. Using a 200-d Ariel 5 observation of the X-ray source the orbital period was estimated to be $\sim 41$ days from the pulse arrival time delays (White et al. 1978). The orbital parameters were in turn refined by Sato et al. (1986) by combining data from Ariel 5, SAS 3, and Hakucho satellites and subsequently by Koh et al. (1997) using BATSE. The orbital period determined from extensive BATSE data is $\sim 41.5$ days and the eccentricity is $\sim 0.46$. The mass of the companion star should be less than $55 M_{\odot}$ in order not to overflow the Roche lobe at periastron. The companion star Wray 977 was classified as of spectral type B2 Ia e (Parkes et al. 1980), already evolved off the main sequence, and is at least $10^{6}$ years old. The P-Cygni type emission line profiles in its optical spectrum show the presence of an extended and expanding atmosphere. Based on a comparison of its optical spectrum with that of $\zeta \mathrm{Sco}$, one of the brightest stars in our galaxy, the companion star was classified to be a B1 Ia+ and the distance was estimated to be $5.3 \mathrm{kpc}$ (Kaper et al. 1995).

Parkes et al. (1980) evaluated the radius of the companion $R_{\mathrm{c}} \sim 0.30 a$ and the Roche radii $R_{\mathrm{L}} \sim 0.64 a$ where $a$ is the semi-major axis of the elliptical orbit. Since Wray 977 
substantially under-fills its Roche Lobe, the dominant mechanism powering the X-ray source for this system is thought to be stellar wind accretion. The mass-loss rate for Wray 977 is in the range of (3-10) $\times 10^{-6} M_{\odot} \mathrm{yr}^{-1}$ (Parkes et al. 1980). The stellar wind velocity measured at a distance of $\frac{r}{R_{\mathrm{c}}} \sim 3$ from the hydrogen Balmer lines is $\sim 300 \mathrm{~km} \mathrm{~s}^{-1}$ (Parkes et al. 1980). This suggests a terminal wind velocity $\left(v_{\infty}\right)$ of $\sim 400 \mathrm{~km} \mathrm{~s}^{-1}$, very low for such a star. This large mass-loss rate from the companion and the unusually low wind velocity result in stagnation of lumps of matter, and a large absorption column density is created along the line of sight. GX 301-2 shows a variable X-ray luminosity in the range $(2-400) \times 10^{35} \mathrm{erg} \mathrm{s}^{-1}$, which depends on the amount of the stellar wind captured. This in turn depends on the density and velocity of the wind and hence provides a good site for probing the wind structure.

A consequence of the high eccentricity and changing orbital separation is a periodically variable accretion rate onto the neutron star. This X-ray binary shows strong periodic X-ray outbursts before most periastron passages. Simple stellar wind models cannot account for the X-ray intensity and absorption column density variations with orbital phase (White \& Swank 1984) nor can tidal mass transfer (Layton et al. 1998). However, as shown by Stevens (1988), Haberl (1991) and Leahy (1991), the dynamical effects of the neutron star on the stellar wind can highly increase the mass-loss rate of the companion toward the neutron star near periastron and may result in a gas stream following it. For GX 301-2 Pravdo et al. (1995) noted a periodic near-apastron flare with lower intensity than the pre-periastron flare and attributed the two flares due to accretion from an equatorially enhanced stellar wind or a circumstellar disk around Wray 977 (also Koh et al. 1997).

In this work we have investigated the spectral variations of the X-ray emission throughout the binary orbit. The column density of absorbing material along the line of sight and flux of the iron emission lines can be valuable for understanding the wind structure in this system. The X-ray spectral lines from the X-ray irradiated wind have been investigated in great detail with the Chandra X-ray observatory (Mukherjee \& Paul 2003; Watanabe et al. 2003). Here we report the spectral analysis of two sets of archival RXTE observations covering two orbital periods of GX 301-2. The observations are described in Sect. 2. We then present the variation of the spectral parameters of the assumed model with orbital phase in Sect. 3 followed by a discussion in Sect. 4 and conclude in Sect. 5.

\section{Archival RXTE observations}

We have used two sets of archival data from the Rossi X-ray Timing Explorer (RXTE) spanning almost the full orbital period of $\sim 41$ days. One set was in 1996 from May 10 to June 15 with seventeen observations while the other set was in 2000 from October 12 to November 19 with thirty-nine observations. The 1996 observations did not cover the phase interval from 0.85 to 0.98 whereas in 2000 the observations had almost a full phase coverage. The time of periastron passage is taken to be the zero of orbital phase (Sato et al. 1986). The useful observation duration in 1996 was $\sim 34 \mathrm{ks}$ and that in 2000 was $262 \mathrm{ks}$.

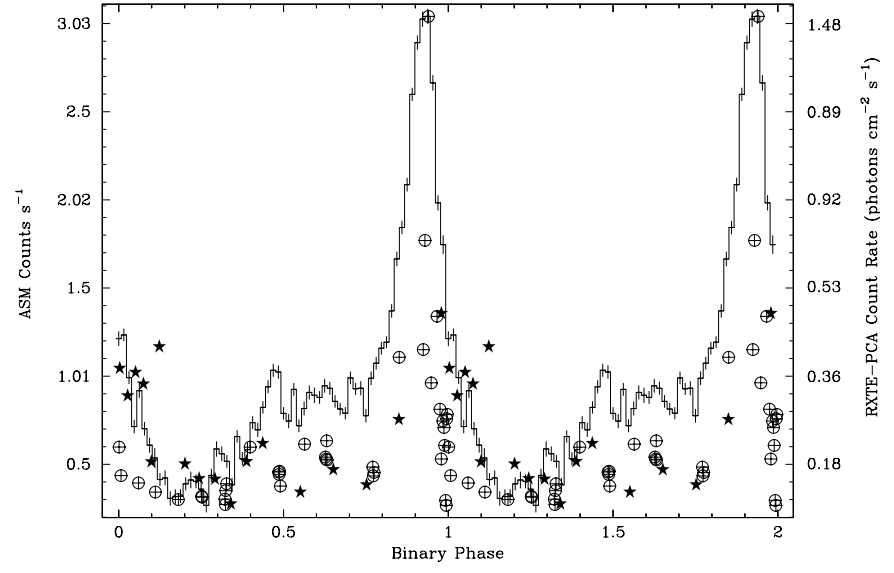

Fig. 1. RXTE-PCA count rate superposed on the RXTE-ASM periodfolded lightcurve as a histogram. The 1996 observations are shown with asterisks and the 2000 observations are shown with marked circles. The binary phase 1.0 corresponds to the periastron passage.

RXTE consists of a large-area proportional counter array (PCA), consisting of five Xenon proportional counter units (PCUs) and sensitive in the energy range of 2-60 keV with an effective area of $6250 \mathrm{~cm}^{2}$ (Jahoda et al. 1996). It also consists of a high energy crystal scintillation experiment (HEXTE; $15-200 \mathrm{keV} ; 1600 \mathrm{~cm}^{2}$ area), and a continuously scanning allsky monitor (ASM; 2-10 keV; $90 \mathrm{~cm}^{2}$ ). A full description of the ASM detector can be found in Bradt et al. (1993). Here we have used the RXTE-ASM long term light curve of GX 301-2 and data from the above mentioned pointed observations with the PCA. The ASM lightcurve folded at the orbital period is shown in Fig. 1 with the PCA count rates superposed on it. On average three PCUs were on for both the observations.

\section{Data analysis and results}

We took the Standard 2 data products of PCA and extracted the source spectra for each dataset using the tool saextrct $v 4.2 \mathrm{~d}$ for the appropriate good time intervals. A systematic error of $1.0 \%$ was added to each spectral bin. Background data files were generated with the tool runpcabackest using background models appropriate for the source brightness and the epoch of the RXTE observation, as provided by the PCA calibration team. The background subtracted source spectra were analyzed with the spectral analysis package XSPEC v 11.2.0 (Shafer et al. 1989). Model spectra were fitted to the observed spectrum for each dataset to determine the important spectral parameters.

\subsection{Choice of spectral model}

The broad band X-ray continuum spectra of accreting pulsars are often found to have the shape of a broken power law or a power law with an exponential cutoff. The break in the spectrum is in the range of $10-20 \mathrm{keV}$, and the power-law photon index below the break energy is in the range of $0-1$ (White et al. 1995). Some pulsars also show cyclotron resonance absorption features, sometimes with more than one harmonics which are usually modeled as multiplicative Gaussian absorption components. In a few pulsars (e.g, Her X-1, Endo et al. 2000), 
observations with good energy resolution CCD detectors showed that the absorption has two components. One component absorbs the entire spectrum while the other component absorbs it partially, fitted by the so called partial absorption model. The partial absorption model is often also described as two different power-law components with the same photon index but different normalisations, being absorbed by different column densities. In a partial absorption model, the second absorption component should have a size smaller than the size of the emission region.

Several observations of GX 301-2 with the CCD detectors of the ASCA satellite clearly showed that the continuum is more complex than a simple absorbed power-law. The ASCA spectra of GX 301-2 in three different orbital phases were fitted satisfactorily with a model consisting of a partially covering power-law component, iron $\mathrm{K} \alpha$ and $\mathrm{K} \beta$ emission lines, and an iron absorption edge at $7.1 \mathrm{keV}$ (Saraswat et al. 1996; Endo et al. 2002). Using the high resolution X-ray grating spectrum of GX 301-2 obtained with the Chandra observatory we found that in addition to the above, there is another broad line component at $6.3 \mathrm{keV}$, which is the Compton backscattered peak of the bright $6.4 \mathrm{keV}$ line (Mukherjee \& Paul 2003; Watanabe et al. 2003). However, the relative intensity of this line is low and it is too close to the $6.4 \mathrm{keV}$ line for inclusion as a separate model component with the moderate resolution RXTE spectrum. Therefore the spectral model that we have chosen to fit the RXTE-PCA spectrum is the same as that of Endo et al. (2002) with the addition of a high energy exponential cutoff. The latter was not required with ASCA spectra due to its limited energy band width.

The analytical form of the model that we have used for spectral fitting is:

$$
\begin{aligned}
N(E)= & \mathrm{e}^{-\sigma(E) N_{\mathrm{H} 1}}\left(S_{1}+S_{2} \mathrm{e}^{-\sigma(E) N_{\mathrm{H} 2}}+G_{1}+G_{2}\right) \\
& E^{-\Gamma} I(E) A(E)
\end{aligned}
$$

where

$$
\begin{aligned}
I(E) & =1 & & \text { for } E<E_{\mathrm{c}} \\
& =\mathrm{e}^{-\left(\frac{E-E_{\mathrm{c}}}{E_{\mathrm{f}}}\right)} & & \text { for } E>E_{\mathrm{c}}
\end{aligned}
$$

and

$$
\begin{aligned}
A(E) & =1 & & \text { for } E<E_{\mathrm{e}} \\
& =\mathrm{e}^{-\tau\left(\frac{E_{\mathrm{e}}}{E}\right)^{3}} & & \text { for } E>E_{\mathrm{e}},
\end{aligned}
$$

$N(E)$ is the intensity (No. of photons s $\mathrm{s}^{-1} \mathrm{keV}^{-1}$ ), $\Gamma$ is the photon index, $N_{\mathrm{H} 1}$ and $N_{\mathrm{H} 2}$ are the two equivalent hydrogen column densities, $\sigma$ is the photo-electric cross-section, $S_{1}$ and $S_{2}$ are the respective normalizations of the power law, $G_{1}$ and $G_{2}$ are the two Gaussian emission lines, $E_{\mathrm{c}}$ is the cut-off energy, $E_{\mathrm{f}}$ the e-folding energy, $E_{\mathrm{e}}$ is the edge energy and $\tau$ is the edge depth.

\subsection{Results}

Apart from a few (seven datasets of 2000 and one of 1996), almost all datasets gave good reduced $\chi^{2}$ between 0.6 and 1.6 for 44 degrees of freedom. The fits with poor reduced $\chi^{2}$ showed

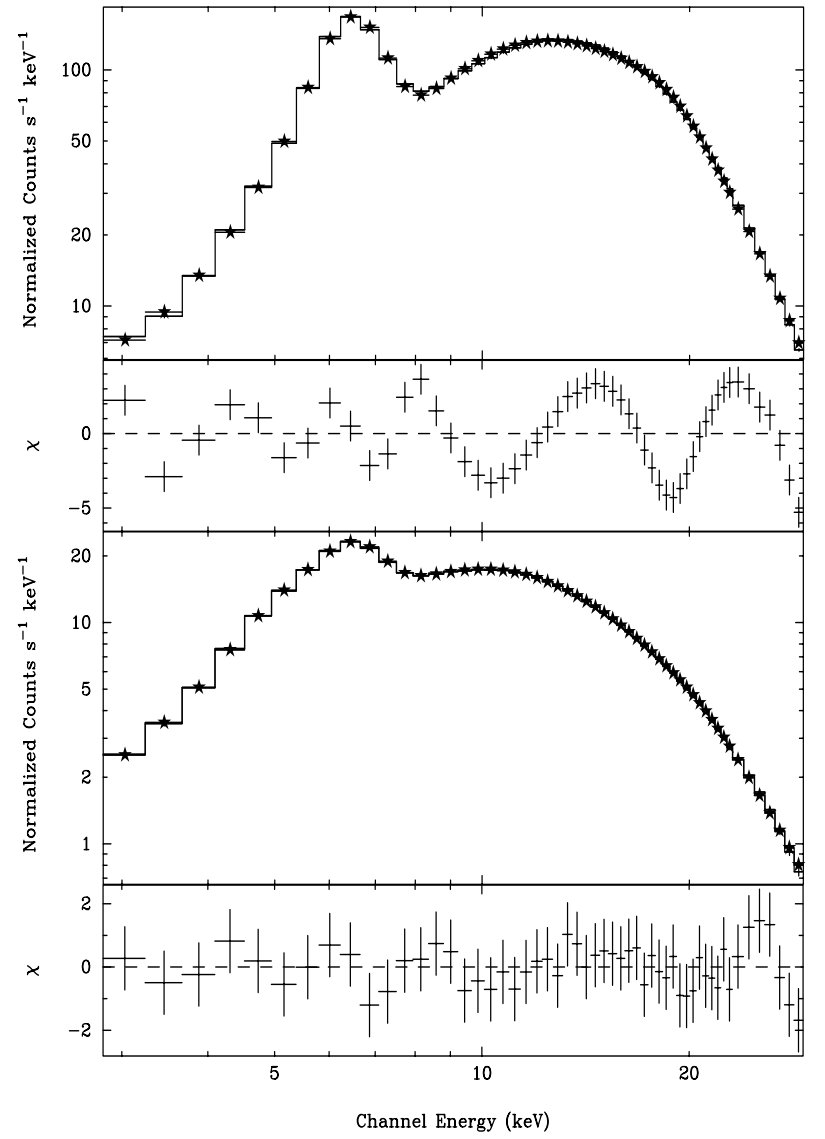

Fig. 2. The top panel shows the worst fit spectra (ObsID: 50066-02-01000 ) with the associated residuals in the second panel. The reduced $\chi^{2}$ came out to be $\sim 7$. The third panel from the top shows one of the many well fitted spectra (ObsID: 50066-01-01-00) along with the residuals in the lowest panel.

wavy residuals with dips around 10, 20 and $30 \mathrm{keV}$. Two representative spectra, one with a poor spectral fit and another with a good spectral fit are shown in Fig. 2. At this stage we are not sure if this particular nature of the residuals in some of the spectra is a systematic phenomenon for this source. Systematic deviations at $\sim 20 \mathrm{keV}$ and $\sim 40 \mathrm{keV}$ were also observed in several broad-band Beppo-SAX spectrum of GX 301-2 (Orlandini et al. 2000).

Ten spectral parameters were varied in the fitting: $N_{\mathrm{H} 1}, N_{\mathrm{H} 2}$, $\Gamma$, the two normalizations, the two iron line intensities, edge depth, $E_{\mathrm{c}}$ and $E_{\mathrm{f}}$. Of these five parameters were kept frozen, the iron-line energies and their FWHM along with the edge energy. For the parameters which were kept fixed we used the values derived from the Chandra HETG spectrum (Mukherjee \& Paul 2003). The variations of the free parameters with orbital phase are shown in Figs. 3 to 6. The 1996 measurements are shown with filled squares and the 2000 data points are shown with open triangles. The error bars shown in the figures correspond to the $90 \%$ confidence levels. For a very few spectra near periastron in which the spectral fits were not very good, the error bars were calculated in XSPEC by increasing the upper limit on the reduced $\chi^{2}$.

The three spectral parameters defining the intrinsic source continuum; photon index, cut-off energy and e-folding energy 


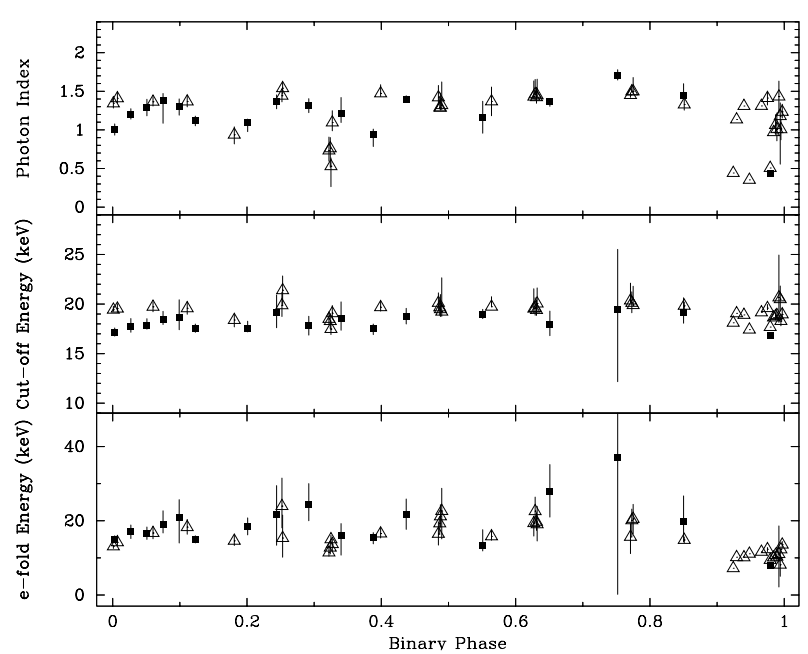

Fig. 3. Variation of the photon index, cut-off energy and e-folding energy with the orbital phase in the upper, middle and lower panels respectively.

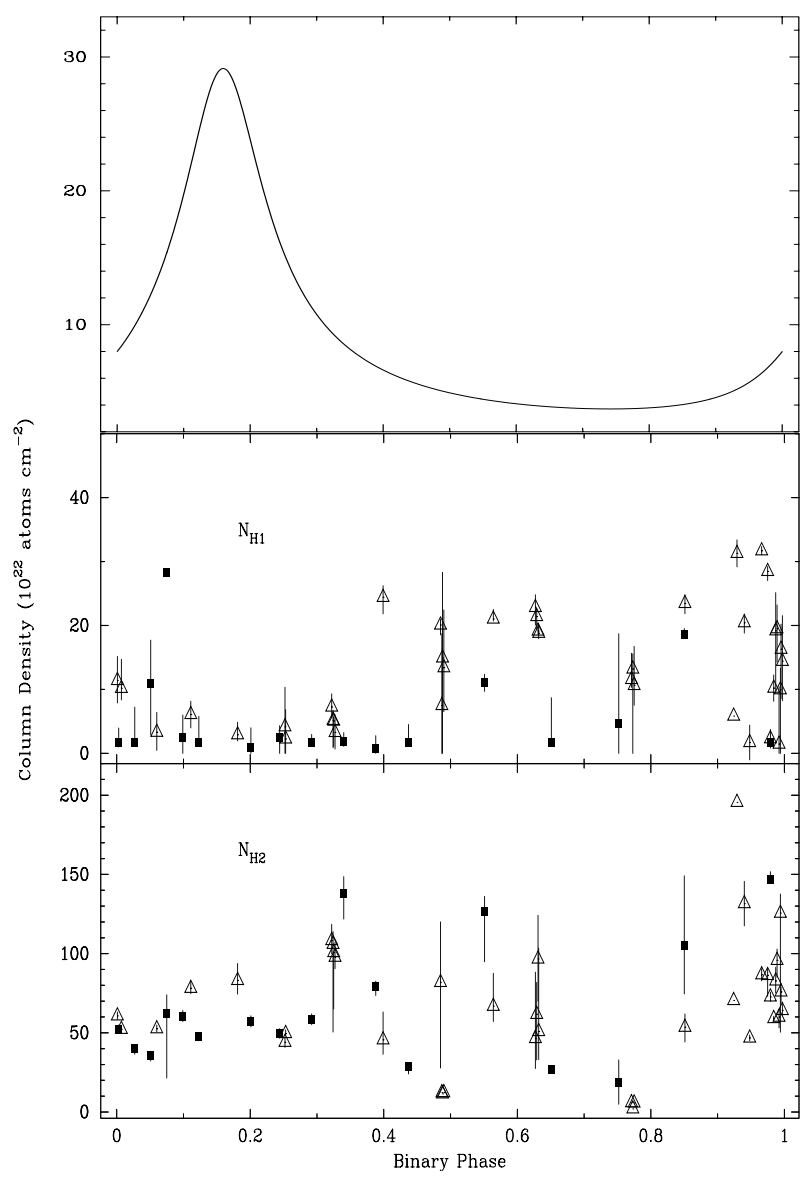

Fig. 4. Variation of column density with the orbital phase. The uppermost panel depicts the model variation, the middle panel showing the observed variation of $N_{\mathrm{H} 1}$ and the lower panel shows the observed variation of $N_{\mathrm{H} 2}$.

are plotted against the orbital phase and shown in Fig. 3. Though the total X-ray luminosity is much higher at the preperiastron phase, there is no remarkable change in the intrinsic continuum spectrum. The cut-off energy varies between

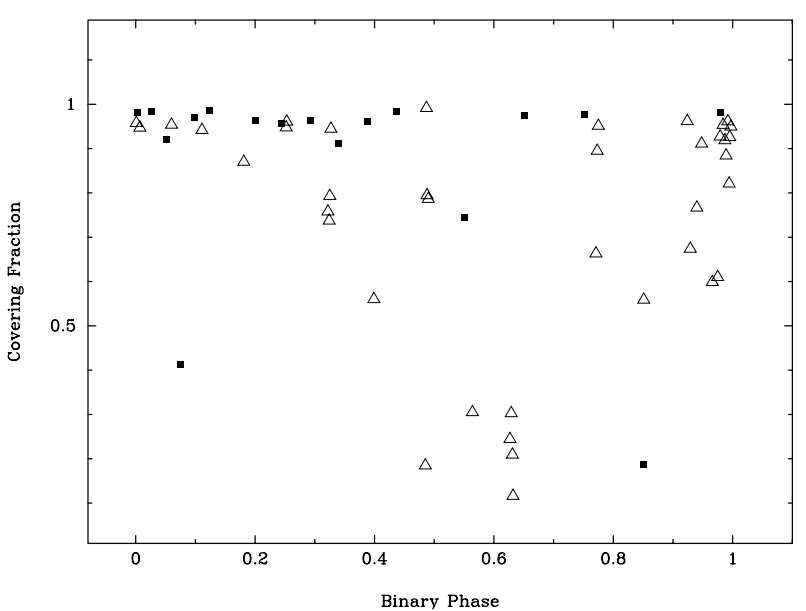

Fig. 5. The variation of the covering fraction of the model with the orbital phase.

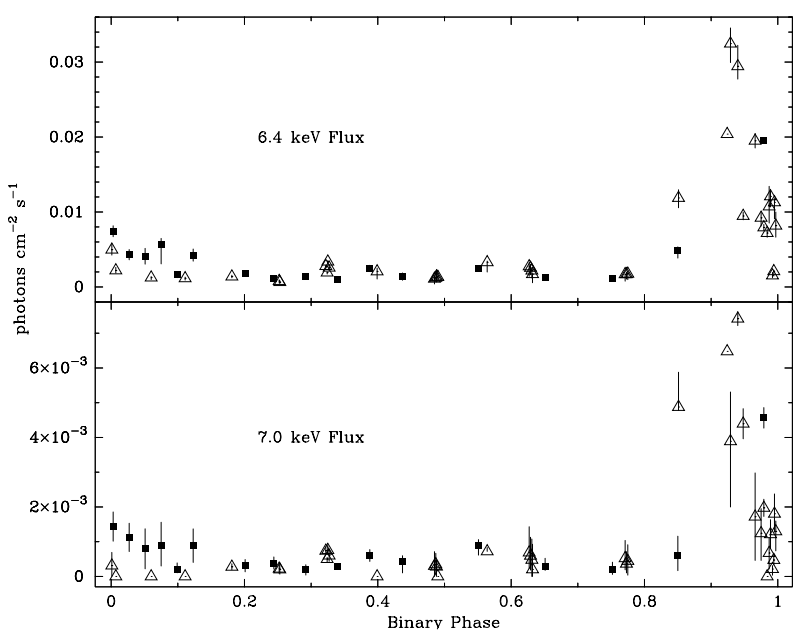

Fig. 6. Variation of the iron-line flux of the model with the orbital phase. Here the upper panel depicts the flux corresponding to the $6.4 \mathrm{keV}$ iron-line and the lower panel shows the $7.0 \mathrm{keV}$ Iron-Line Flux respectively.

$17-22 \mathrm{keV}$ and the e-folding energy is about $20 \mathrm{keV}$ except at a few points where the spectral fits are poor. The variation of the photon index with phase shows that $\Gamma$ in general lies between 1.0-1.5 for both sets of observations while some values drop below one. These values for $E_{\mathrm{c}}, E_{\mathrm{f}}$ and photon index are more or less consistent with the previously measured values in some binary phases reported by other authors (White et al. 1983; Orlandini et al. 2000).

The changes of the two column densities with orbital phase for both observations are plotted in Fig. 4 (second and third panels). The figure shows a considerable increase in column density near periastron; but also a substantial scatter in the values in the intermediate phases. We have also depicted the variation of the covering fraction measured from our model in Fig. 5. From Fig. 6 we find that the iron-line intensities are much larger than average before the periastron passage and show a small increase near phase $\sim 0.1$ for 1996 . Though the 2000 set shows a steep rise near periastron, no such rise near phase $\sim 0.1$ is noticeable. 


\section{Discussion}

\subsection{The spectrum}

The X-ray spectrum of GX 301-2 is characterised by a heavy and variable photoelectric absorption with a strong iron $\mathrm{K} \alpha$ emission line (White \& Swank 1984). High resolution observations with $\mathrm{X}$-ray gratings helped the discovery of a Compton backscattered peak at $\sim 6.3 \mathrm{keV}$ in the spectrum (Mukherjee \& Paul 2003; Watanabe et al. 2003). From detailed MonteCarlo simulations and comparisons with the observed spectra, Watanabe et al. (2003) constrained the physical properties of the cold fluorescing medium surrounding the neutron star. In our analysis of the low energy resolution RXTE-PCA spectrum covering almost the entire binary orbit, the $2-30 \mathrm{keV}$ $\mathrm{X}$-ray spectrum is modeled using a partially covering high energy cut-off power-law component, two emission lines at 6.4 and $7.0 \mathrm{keV}$, and one absorption edge at $7.1 \mathrm{keV}$ due to neutral iron. The Compton backscattered peak was not included in this model as the RXTE proportional counters with moderate energy resolution cannot separate it from the fluorescence line. We found that this model provides good fits for most datasets throughout the orbit save for a very few at energies above $8 \mathrm{keV}$. The reason why a few datasets do not follow the general trend remains to be explored. The average values of the free parameters measured here over the full binary orbit; viz. photon index, e-folding energy and cut-off energy follow the general trend which were earlier measured only in some phases of the binary period (White et al. 1983; Orlandini et al. 2000).

\subsection{Variations in column density}

The equivalent hydrogen column density of material that absorbs the primary X-ray emission is found to be quite high. At the same time, a large variation of the column density throughout the binary orbit (from $10^{22}$ to $10^{24}$ atoms $\mathrm{cm}^{-2}$ ) seems to be one of the characteristics of the X-ray spectrum of GX 301-2. The large variation of the column densities $N_{\mathrm{H} 1}$ and $N_{\mathrm{H} 2}$ at all orbital phases indicate clumpiness of the stellar wind at different size scales.

In the partial covering model used to fit the X-ray spectrum of many accreting pulsars, $N_{\mathrm{H} 2}$ is interpreted as the column density of the material local to the X-ray source, while $N_{\mathrm{H} 1}$ accounts for the rest of the material (along with galactic absorption). The covering fraction is defined as Norm2/(Norm1+Norm2) where Norm1 and Norm2 are respectively the normalizations of the two power-laws. It is seen from Fig. 5 that the covering fraction remains substantially high almost throughout the orbit which means that there is dense and clumpy material present throughout. Only a small fraction of the primary X-rays come out without facing this dense and clumpy material. The presence of a dense cloud around the neutron star and close to it is also supported by the strong Compton recoil component detected with the Chandra grating spectrum and its successful reproduction by Monte Carlo simulations (Watanabe et al. 2003).
To compare our measured values of column densities, we have obtained a model variation of column density with the orbital phase using a spherically symmetric wind emanating from the companion star Wray 977. The stellar wind had a Castor et al. (CAK, 1975) velocity profile :

$v_{\text {wind }}=v_{\infty} \sqrt{1-\frac{R_{\mathrm{c}}}{r}}$

where $v_{\infty}$ is the terminal velocity for the stellar wind of Wray $977\left(400 \mathrm{~km} \mathrm{~s}^{-1}\right), R_{\mathrm{c}}$ is the radius of Wray 977 and $r$ is the distance from it.

The column densities were evaluated by numerical integration along the line of sight from the neutron star to the observer at infinity as the neutron star traversed the elliptical orbit. The observer was on a diferent plane than the orbit and the inclination angle was taken to be $69^{\circ}$. The projected line of sight made an angle of $63^{\circ}$ with the major axis of the ellipse (Fig. 6 of Pravdo \& Ghosh 2001, PG from hereon).

The assumed mass-loss rate was $10^{-5} M_{\odot} \mathrm{yr}^{-1}$. The result was a smooth curve with a peak between phases 0.1 and 0.2 with no rise near periastron (First panel in Fig. 4). The values of the column densities were of the order of $10^{22}$ to $10^{23}$ atoms $\mathrm{cm}^{-2}$ The peak between phases 0.1 and 0.2 was expected since the line of sight passes through the densest parts of the wind during these phases. On the other hand, our measured column densities did not show such a smooth variation and moreover the values also varied widely from $10^{22}$ to $10^{24}$ atoms $\mathrm{cm}^{-2}$. Thus it is clear that the observed variation in column density cannot be explained by a spherically symmetric CAK wind only.

Two models have been put forward to explain the orbital modulation of the column density of GX 301-2. Leahy (1991) fitted the observed variation in column density versus orbital phase (from TENMA data) with a spherically symmetric stellar wind and a linear gas stream. The peak near periastron was fitted when the gas stream was introduced (Fig. 3, Leahy 1991). The model with only a CAK wind component was found to be unacceptable as it could not fit the rise in the column densities at pre-periastron. A gas stream can be due to the dynamical effect of the neutron star on the companion wind and the effect would be strongest at periastron when the neutron star is closest to the companion. Leahy (2002) also explained the flux peak prior to periastron and a broad peak near apastron in the RXTE-ASM lightcurve with the same model. However, we note that this is a very simplistic model since it does not include the viscous time scale of gas flow through the accretion disk which is likely to cause a few days delay between the interaction of the neutron star with the gas stream and the resultant X-ray enhancement. PG on the other hand, have proposed the existence of an equatorially enhanced circumstellar disk about Wray 977 which can also adequately explain the orbital flux modulation of the RXTE-ASM lightcurve. The proposed model describes two interactions of the neutron star with the disc which gives rise to the two peaks in the column density, one at pre-periastron and the other between phases 0.1 and 0.2 , with a smooth component elsewhere. However, the orbital dependence of the absorption column density expected from such a model (PG, Fig. 7b) or for that matter from the gas 


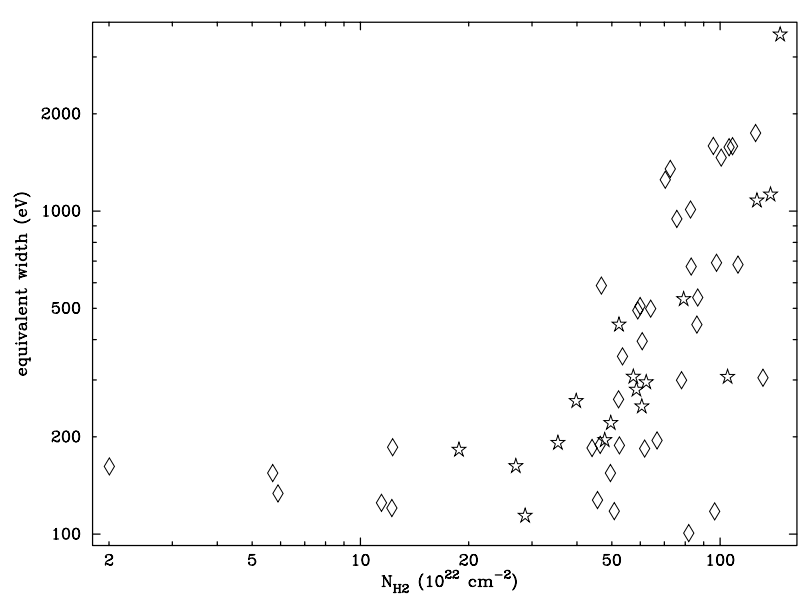

Fig. 7. Correlation of equivalent width of the $6.4 \mathrm{keV}$ iron-line with $N_{\mathrm{H} 2}$. Both the axes are in logarithmic scale. The stars denote the 1996 dataset and the diamonds represent the 2000 dataset.

stream model of Leahy (1991) is very different from the variation of the column densities at different orbital phases with the RXTE-PCA data as measured by us (Fig. 4, second and third panel). From the observed variation of the column density as measured by us it appears that there are probably strong inhomogeneties in the wind that are causing large fluctuations in the column densities. This can be supported by the fact that almost throughout the binary orbit the covering fraction remains high (Fig. 5) which indicates the presence of clumpy inhomogeneous material.

A possible reason for the increase in column densities near periastron may be that very strong X-ray emission at the preperiastron phase ionises most of the X-ray wind near the neutron star. This in turn can reduce the rate of acceleration of the wind by the UV emission causing the wind velocity profile to differ from that of an isolated star. An observable effect of this is additional clumping of material at the pre-periastron position and an increase in the measured column densities.

The RXTE-ASM lightcurve of another very similar high mass X-ray binary pulsar 4U 1907+09 was also modeled using a spherical wind model (Roberts et al. 2001). A phase-locked secondary flare in the lightcurve was reported along with a preperiastron flare. Though the simple wind model fitted the timeaveraged lightcurve, it failed to account for the secondary flare and also for the variation of the absorption column density with orbital phase. A spherical wind plus gas stream in turn fitted the variation in column density.

\subsection{Variation of iron-line flux}

The two iron lines included in our model show large increases in flux near periastron and a possible small increase near phase 0.1 (at least for 1996). The peak near periastron (phase $\sim 0.9$ ) is not very evident in the 1996 data due to the lack of enough observations, though an increasing trend can possibly be inferred. The average magnitude of the $7.0 \mathrm{keV}$ iron-line flux is about an order of magnitude less than that of the $6.4 \mathrm{keV}$ iron-line flux. The fluxes for both lines in the intermediate phases show a more or less steady value. We also found that the iron-line equivalent width has a clear correlation with $N_{\mathrm{H} 2}$ with considerable scatter, as shown in Fig. 7. The correlation indicates that the hard X-rays are reprocessed by the material local to the hard X-ray source and the neutron star interacts with an enhanced mass near periastron, but the presence of enhanced mass near phase 0.1 as proposed by PG is not very clearly seen in the line fluxes. Previously Makino et al. (1985) and Endo et al. (2002) have showed that such a correlation exists in GX 301-2 from observations made in certain orbital phases. Endo et al. (2002) discusses that a simple relation of the equivalent width exists with the line of sight column density as long as the fluorescing plasma is optically thin and fully surrounds the pulsar. For that case the equivalent width increases monotonically with the column density. The scatter seen in our correlation may be due to the clumpiness of the reprocessing material. However, for small values of $N_{\mathrm{H} 2}\left[(2-30) \times 10^{22}\right.$ atoms $\left.\mathrm{cm}^{-2}\right]$, the equivalent width appears to be nearly constant. This may arise if at low values of $N_{\mathrm{H} 2}$ the clumpy absorber does not completely surround the neutron star. Another possibility is that a fraction of the iron line may be produced by the absorbing material associated with $N_{\mathrm{H} 1}$, which becomes the dominant component for small values of $N_{\mathrm{H} 2}$.

\section{Conclusions}

1. The partial covering power-law model with two emission lines and an absorption edge describes the X-ray spectrum of GX 301-2 well throughout the entire binary orbit.

2. The column densities measured are very high with a large variation throughout the binary orbit indicating a clumpy nature of the stellar wind.

3. In this work we have measured the variation of column density with orbital phase and compared it with the variation expected in the disk wind model of PG and also in the gas stream model of Leahy (1991).

4. The correlation of the iron-line equivalent width with the column density $\left(N_{\mathrm{H} 2}\right)$ suggests that most of the iron line is produced by the local clumpy matter surrounding the neutron star.

Acknowledgements. Our work has made use of the data obtained from the High Energy Astrophysics Archive Research Center (HEASARC) provided by NASA/GSFC. We express immense gratitude towards the anonymous referee for greatly improving our work. It is a pleasure for U.M. to thank K. Garai for his help in wind modeling. He also acknowledges the Kanwal Rekhi Scholarship of T.I.F.R. Endowment Fund for partial financial support.

\section{References}

Bradt, H. V., Rothschild, R. E., \& Swank, J. H. 1993, A\&AS, 97, 355

Castor, J. I., Abbott, D. C., \& Klein, R. I. 1975, ApJ, 195, 157

Endo, T., Nagase, F., \& Mihara, T. 2000, PASJ, 52, 223E

Endo, T., Ishida, M., Masai, K., et al. 2002, ApJ, 574, 879

Haberl, F. 1991, A\&A, 252, 272

Jahoda, K., Swank, J. H., Giles, A. B., et al. 1996, in EUV, X-ray and Gamma-Ray Instrumentation for Astronomy VII, ed. O. H. W. Siegmund, \& M. A. Gummin, SPIE, 2808, 59 
Kaper, L., Lamers, H. J. G. L. M., Ruymaekers, E., et al. 1995, A\&A, 300, 446

Koh, D. T., Bildsten, L., Chakrabarty, D., et al. 1997, ApJ, 479, 933

Layton, J. T., Blondin, J. M., Owen, M. P., \& Stevens, I. R. 1998, New Astron., 3, 111

Leahy, D. A. 1991, MNRAS, 250, 310

Leahy, D. A. 2002, A\&A, 391, 219

Makino, F., Leahy, D. A., \& Kawai, N. 1985, Space Sci. Rev., 40, 421

Mukherjee, U., \& Paul, B. 2003, BASI, 31, 369

Orlandini, M., dal Fiume, D., Frontera, F., et al. 2000, Adv. Space Res., 25, 417

Parkes, G. E., Culhane, J. L., Mason, K. O., \& Murdin, P. G. 1980, MNRAS, 191, 547

Pravdo, S. H., Day, C. S. R., Angelini, L., et al. 1995, ApJ, 454, 872

Pravdo, S. H., \& Ghosh, P. 2001, ApJ, 554, 383
Roberts, M. S. E., Michelson, P. F., Leahy, D., et al. 2001, ApJ, 555, 967

Saraswat, P., Yoshida, A., Mihara, T., et al. 1996, ApJ, 463, 726

Sato, N., Nagase, F., Kawai, N., et al. 1986, ApJ, 304, 241

Shafer, R. A., Haberl, F., \& Arnaud, K. A. 1989, XSPEC: An X-ray Spectral Fitting Package, ESA TM-09 (Paris: ESA)

Stevens, I. R. 1988, MNRAS, 232, 199

Watanabe, S., Sako, M., Ishida, M., et al. 2003, ApJ, 597, L37

White, N. E., Mason, K. O., Huckle, H. E., Charles, P. A., \& Sanford, P. W. 1976, ApJ, 209, L119

White, N. E., Mason, K. O., \& Sanford, P. W. 1978, MNRAS, 184, 67

White, N. E. Nagase, F., \& Parmar, A. N. 1995, in X-ray Binaries, ed. W. H. G. Lewin, J. van Paradijs, \& E. P. J. van den Heuvel (New York: Cambridge Univ. Press), 1

White, N. E., Swank, J. H., \& Holt, S. S. 1983, ApJ, 270, 711

White, N. E., \& Swank, J. H. 1984, ApJ, 287, 856 